\title{
Index des personnes
}

Adam de la Halle (poète et chanteur, prince du puy d'Arras) 25-26

Adamson, John (messager) 75

Adenet le Roi (poète, roi des ménestrels) 24, 27-28, 42, 163

Ams, Rúdin (cordonnier de Zurich) 103

Angleterre, roi d'

- Édouard I ${ }^{\text {er }} 33,40^{+92}$

- Édouard II 33, 37

- Édouard III 33, 38, 41

- Henri IV 33, 71, 75

- Henri V 33

- Henri VIII 69

- Jacques II (James II) 67, 76

- Richard I ${ }^{\text {er }}$ (Cœur de Lion) $159^{17}$

- Richard II 33

Arran, comte d'

- James Stewart of Bothwellmure 73

Atlas, Allan W. (musicologue) 31

Bakhtin, Mikhail (historien et théoricien de la littérature) 131

Barril, Jean (délinquant) 148

Baugé, Pierre de, alias Pierre V (roi de la basoche) 119

Bavière, duc de

- Albert II 30, 40, 46

Bercé, Yves-Marie (historien) 131

Berry, duc de

- Jean 14, 85

Bloch, Marc (historien) $12^{11}$

Bodel, Jean (poète, prince du puy d'Arras) 25

Boisard, Jean, dit Verdelet (roi des ménestrels) 27, 32, 34, 37

Bosenton, Beaul [Veaul] de (maître d'hôtel) 50-51

Bourdieu, Pierre (sociologue) 139

Bourgogne, duc de

- Charles le Téméraire $49-51^{+39,40}, 57^{+70}$, 92

- Jean sans Peur 32, 46, 86

- Philippe le Bon $32,34,49,85^{21}-86^{29}$, 90, 92

- Philippe le Beau 50, 52-53

- Philippe le Hardi 27, 46

Bourré, Jean (maître des comptes) 149

Bouvet, Honoré (écrivain) 70

Brabant, duc de
- Henri III 27-28

Bretel, Jacques (ménestrel) 45

Bretel, Jean (poète, prince du puy d'Arras) 25

Brisebarre, Jean [dit Jean Le Court] (ménestrel) 41-42

Bubenberg, Adrian II de (noble, roi des chaudronniers de Berne) 101

Burnett, Charles (historien) 73

Charmillon, Jean (roi des jongleurs) $36^{+67}$

Chartier, Jean (chroniquer) 148

Chartier, Roger (historien) 153

Caresme, Jean (roi des ménestrels) 32, 34

Caumez, Jehan (roi des ménestrels) 27, 32

Caveron, Robert (roi des ménestrels) 27, 32

Clarence, duc de

- Thomas de Lancastre 52, 54, $55^{59}$

Condé, Jean de (roi des ménestrels) 28 29,34

Coppin de Brequin (roi des ménestrels) $27,32,35^{63}$

Costeley, Guillaume (musicien) 128

Courolles, Jean de (hôtelier, prince d'amours et prince des arbalétriers) 140

Cumming of Inverallochy, Sir William (héraut Marchmont) $65^{+8}, 70-72,79$

Dahhaoui, Yann (historien) 18

Damien, Pierre (théologien) 155

Dannhauer, Johann Conrad (théologien et philologue) 125

Davis, Natalie Zemon (historienne) $131^{+2}$

Dickinson, William Croft (historien) 76

Dobozy, Maria (philologue) 30

Dragonetti, Roger (philologue) 25

Durham of Largo, Sir Alexander (roi d'armes Lyon) 67

Ebersdorf, Pierre de (avocat légal de la confrérie des ménestrels à Vienne) 35

Écosse, reine d'/roi d'

- Jacques III [James III] $67^{18}, 70$

- Jacques IV [James IV] $67^{18}, 70^{31}-71$, 73,79

- Jacques V [James V] 66-67, 69, 72

- Marie de Guise 67

- Robert I ${ }^{\text {er }}$ the Bruce 66 
- Robert III 68

Egenolff, Christian (imprimeur) 124

Facien, Jean (roi des ménestrels) 27, 32, 37

Fenlon, Iain (musicologue) 31

Flandre, comte de

- Gui II de Dampierre 27

Forman of Luthrie, Sir Robert (roi d'armes Lyon) $65^{8}-66,73$

France, roi de

- Charles VI 14, 48, 85

- Charles VII 14, 26, 35, 140

- Charles VIII 35, 53

- François I ${ }^{\text {er }} 153$

- Henri II 32

- Jean II (le Bon) 157

- Louis VIII 159

- Louis IX (Saint Louis) 83, 159

- Louis XI 35, 50, 92, 161

- Louis XII 35

- Philippe Auguste (Philippe II) 82, $158^{15}-159$

- Philippe III 159

- Philippe IV (le Bel) 2923, 32, 36, 45, 114

- Philippe V (le Long) 26, 32, 84

Francolin, Jean de (roi d'armes d'Hongrie) 49

Frauenlob, voir Heinrich von Meissen

Froissart, Jean (chroniqueur) 29, 82, 156

Grant, Francis (héraldiste, roi d'armes Lyon) 74,78

Grass, Nikolaus (historien et folkloriste) 11

Grinberg, Martine (historienne) 132

Guidobaldi, Nicoletta (historienne) 31

Gvozdeva, Katja (philologue) 144

Habert, François (poète, prince de la Basoche) 117-118

Hainaut, comte de

- Guillaume $\mathrm{I}^{\mathrm{er}}$ le Bon $29^{+23}$

- Guillaume IV de Bavière 32, 34

Hannelet, Jean (roi des ménestrels) 32, 34

Haquin, Gilles (prévôt de Paris) 26

Heinrich von Meissen, dit Frauenlob (chanteurs de minne) 30

Helttampt, Wolfhart (maître des comptes) 30

Hepburn, Patrick (shérif de Berwick) 75

Hugo, Victor (écrivain) 11, 153, 161

Huizinga, Johan (historien) 49
Isaac, Thomas (roi d'armes Toison d'or)

$$
51,54-56^{+64,66}, 58
$$

Jean le Bel (chroniquer) 29

Jean le Court, voir Brisebarre, Jean

Jeanne d'Arc, dit la Pucelle d'Orléans 93

Jeanne de Valois, épouse du comte Guillaume $\mathrm{I}^{\mathrm{er}}$ de Hainaut $29^{+23}, 34$

Kintyre (poursuivant d'armes), voir Loutfut, Adam

La Marche, Olivier de (chroniqueur) 4950

La Vigne, André de (poète) 119

Lacroix, Paul (écrivain, historien) 82

Lefèvre de Saint-Rémy, Jean (roi d'armes Toison d'or, chroniqueur) $46^{18}, 54$

Le Franc, Martin (clerc et poète) 34

Lindenzwig (cordonnier de Mulhouse) 103

Lindsay of the Mount, Sir David (roi d'armes Lyon) 66, 72, 74 ${ }^{+61}, 77,79$

Lyon (roi d'armes), voir Durham of Largo, Sir Alexander; Forman of Luthrie, Sir Robert; Grant, Francis; Lindsay of the Mount, Sir David; Pettigrew, Thomas; Stewart, Sir William; Thomson of Keillour, Henry

Loutfut, Adam (poursuivant d'armes Kintyre) 67-68, 70-72, 78

Marchmont (héraut), voir Cumming of Inverallochy, Sir William; Meldrum, John

Meldrum, John (héraut Marchmont) 75

Miraulmont, Pierre de (juriste) $114^{4}$, 116

Morlee, William de (ménestrel, roy de North) 38

Muchembled, Robert (historien) 11, 131

Orcades, comte des

- William Sinclair 70

Ormont, Guillaume (prévôt de Paris) 26

Offenburg, Henman (magistrat et diplomate, seigneur des compagnons potiers) $108^{+37}$

Pasquier, Étienne (écrivain et juriste) 11 , 80

Pettigrew, Thomas (roi d'armes Lyon) 6566 
Philippa de Hainaut, reine d'Angleterre $29^{23}, 41-42$

Pleij, Herman (philologue) 135, 139

Poitevin, Hennequin (roi des ménestrels) 27,32

Portevin, Jehan (roi des ménestrels) 27, 32

Rebecques, Gilles de (roi d'armes de Hainaut) 48

Reid, Dylan (historien) 139

Rossiaud, Jacques (historien) 131

Rothesay (héraut) 68

Salenone, Antoine de (chambellan) $50-51^{+39}$

Savary de Beaulieu, dit l'abbé des malprofitants $150-151$

Shrewsbury, comte de

- John Talbot 68

Soleuvre, voir Salenone, Antoine de

Soummeillon, Robert (poète, prince du puy d'Arras) 25

Stevenson, John H. (historien) $65^{7}, 68^{+25}, 76$

Stewart, Sir William (roi d'armes Lyon) 66

Terrier, Étienne (délinquant) 148

Thomson of Keillour, Henry (roi d'armes Lyon) 65
Toison d'or (roi d'armes), voir Isaac, Thomas; Lefèvre de Saint-Rémy, Jean Toulongeon, Claude de (conseiller et chambellan) 50-51

Trazegnies, Jean II de (chambellan) 50

Tudor, Marguerite (reine consort d'Écosse) $67^{18}, 69^{28}, 71,79$

Urgel, comte d'

- Ermengaud I ${ }^{\text {er }} 155$

van Anrooij, Wim (philologue) 49

Vauldrey, Louis de (chambellan) $51^{+40}$

Verdelet, voir Boisard, Jean

Versoris, Nicolas (avocat) 149

Waldmann, Hans (bourgmestre, roi des chaudronniers de Zurich) 101

Walther von der Vogelweide (chanteur de minne) 30

Werner (seigneur et protecteur de l'association des compagnons cordonniers) 103,108

Wolfger de Erla (évêque de Passau) 30

Zumthor, Paul (médiéviste) 41 